\title{
Pollution de l'air : méthodes de mesure et impact sur notre environnement
}

\author{
B. Calpini
}

École Polytechnique Fédérale de Lausanne, CH-1015 Lausanne, Switzerland

\begin{abstract}
La conférence GACHI (Groupe d'Analyse Chimique Interdisciplinaire) organisée en octobre 1998 à l'École Polytechnique Fédérale de Lausanne a porté sur le thème "Pollution de l'air : Méthodes de mesure et impact sur notre environnement », et donné lieu à la parution d'un dossier spécial dans la revue EurJAC. Cet article est un résumé de ce dossier, et souligne quelques-uns uns des aspects importants présentés en détail dans le dossier EurJAC.
\end{abstract}

es activités humaines ont de tout temps donné lieu à des effets de pollution de l'air. Sans doute que nos ancêtres n'ont pas considéré comme telle la pollution induite par leur feu de bois ! Cependant au $\mathrm{xvIII}^{\mathrm{e}}$ siècle à Londres, lorsque la population se mit à utiliser de manière intensive le charbon à haute teneur en soufre à la place du bois, la détérioration de la qualité de l'air fut ressentie comme une véritable nuisance, surtout à basse altitude en raison de conditions météorologiques particulières. Avec une population atteignant six milliards d'individus sur terre et une consommation croissante des ressources naturelles et énergétiques, le phénomène de pollution de l'air a pris aujourd'hui une ampleur sans précédent : il n'affecte plus notre atmosphère terrestre de manière localisée, mais au contraire génère des changements à l'échelle de la terre entière.

\section{En pollution de l'air, que devons-nous mesurer?}

Dans cet immense « réacteur chimique » qu'est notre atmosphère terrestre, il s'agit de définir nos besoins en terme de mesure de pollution de l'air, à savoir quel type de mesure, sur quelle extension spatiale et temporelle, à quelle fréquence, et enfin avec quelle précision. Pour répondre à ces différentes questions, rappelons de quoi est fait ce réacteur atmosphérique. Il est caractérisé par la présence des émissions primaires qui sont soit d'origine naturelle (par exemple les hydrocarbures émis par les forets), soit produites par l'homme (émissions de type industrielles, trafic, ....). À ces émissions primaires vient s'ajouter la chimie réactionnelle en partie induite par le soleil, les effets de la météorologie qui impliquent le transport et la diffusion des polluants dans l'atmosphère, et enfin les phénomènes de déposition par voie sèche (contact gaz-surface terrestre) ou par voie humide (condensation de gaz à la surface de gouttelettes). Donc, mesurer la pollution de l'air dans ce réacteur atmosphérique, c'est mesurer les espèces chimiques présentes dans l'atmosphère et les paramètres météorologiques de ce réacteur.

Quand effectuer de telles mesures ? Il y a en fait trois champs d'application de la mesure de pollution de l'air bien distincts, à savoir :

$1^{\circ}$ les mesures visant le contrôle de cote d'alerte de la pollution de l'air ;

$2^{\circ}$ les mesures donnant une information à long terme de l'évolution de la pollution de l'air ;

$3^{\circ}$ l'étude spécifique d'épisodes photochimiques particuliers.

\section{Les mesures de cote d'alerte}

Les ordonnances en matière de qualité de l'air sont, encore aujourd'hui, définies par chaque pays individuellement, et tendent à fixer les critères de maxima de pollution au-delà desquels une politique active de réduction de cette pollution doit être appliquée. Ces maxima sont définis soit en limite de concentration, ou de manière plus pertinente en « dose limite d'exposition » correspondant au produit de la concentration par le temps d'exposition des individus. Nous verrons plus loin que ce critère de dose est de fait directement lié à la santé des individus ainsi qu'à l'impact de la pollution de l'air sur notre environnement naturel, par exemple sur les rendements agricoles.

Ces cotes d'alerte sont définies sur les « gaz critères » ou de référence que sont les oxydes d'azote $\left(\mathrm{NO}+\mathrm{NO}_{2}=\mathrm{NO}_{x}\right)$, le dioxyde de soufre $\mathrm{SO}_{2}$, le monoxyde de carbone $\mathrm{CO}$, l'ozone $\mathrm{O}_{3}$ et les particules en suspension dans l'air, les $\mathrm{PM}_{10}$ qui sont les particules plus petites que 10 micromètres de diamètre moyen. Dans ce premier champ d'application qu'est la mesure de cote d'alerte, les stations de mesure sont situées au cœur des sources d'émission primaires les plus intenses. Notons que cette mesure de cote d'alerte a longtemps représenté le critère central de définition de l'établissement d'un réseau de mesure de la qualité de l'air 
pour une agglomération ou une région donnée. À l'expérience, il s'avère que les bases de donnée ainsi collectées ne sont pas utilisées, si ce n'est bien sûr pour annoncer ces situations d'alarme à la pollution de l'air.

\section{La mesure de tendance}

Alors que la cote d'alerte est liée à une mesure de pic de pollution se développant sur quelques heures, la mesure des tendances ( "trend measurements ») est obtenue à partir d'une série temporelle sur plusieurs années ou décennies. Il s'agit alors de mesurer ces gaz de référence, mais avec une instrumentation dont la maintenance et le renouvellement n'affecte pas l'analyse des résultats sur le long terme. Présentons ici deux exemples issus de la base de donnée du réseau de surveillance de la qualité de l'air de la ville de Lausanne (Baehler EurJAC) d'observations de longue durée en atmosphère urbaine. La figure 1 montre l'évolution de la concentration de $\mathrm{SO}_{2}$ en ville de Lausanne sur les trente dernières années, et met en évidence une réduction remarquable de ces émissions, soulignant par là l'efficacité et la réussite des nouvelles normes imposées en matière d'émissions dues aux chauffages des bâtiments en particulier.

La figure 2 montre l'évolution de 1975 à 1995 du dioxyde d'azote, toujours sur la même agglomération, proche d'une voie de circulation à grand trafic.

La limite légale en Suisse pour le $\mathrm{NO}_{2}$ y est indiquée pour une valeur de 30 microgramme $/ \mathrm{m}^{3}$. Dans ce deuxième cas, et bien que ces valeurs à l'immission soient ici principalement dues au trafic et encore clairement au delà des normes, une réduction sensible est mesurée dès 1983, associée principalement à la mise en place de catalyseurs sur les échappements de voitures. Cette réduction est d'autant plus remarquable que le nombre de véhicules a fortement augmenté durant cette même période à proximité de ce site de mesure.

Aussi bien pour la détermination de cote d'alerte que pour les mesures de tendances à long terme, l'expérience montre que ce n'est pas tant le nombre de stations de mesures qui importe, mais bien la qualité des sites de mesure, c'est-à-dire aussi bien du point de vue du choix de l'emplacement du site que de la parfaite maintenance et du calibrage des appareils. Partant de ce constat, les administrations

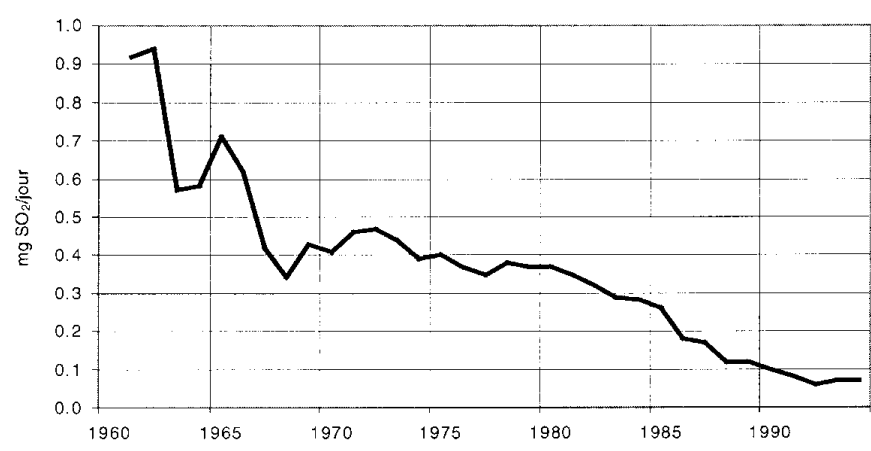

Figure 1. Évolution de la concentration de dioxyde de soufre sur les trente dernières années au centre ville de Lausanne (Baehler EurJAC).

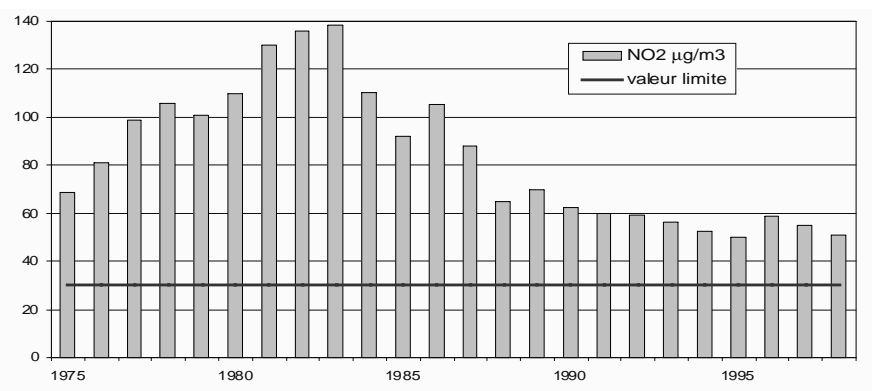

Figure 2. Évolution de la concentration de dioxyde d'azote de 1975 à 1995 proche d'une rue à forte densité de trafic au centre ville de Lausanne (Baehler EurJAC).

publiques responsables des différents réseaux de mesure de la qualité de l'air en Suisse ont réduit depuis peu le nombre de ces installations ces dernières années, ce qui est résumé dans le tableau I.

Notons enfin que cette réduction est aussi le résultat de la prise de conscience des agences de protection de l'environnement que les bases de données générées par «trop » de stations de mesure n'ont de fait pas fait l'objet d'une analyse qui les valorise. Ce point nous amène au troisième champs d'application des mesures de pollution de l'air.

Tableau I. Diminution du nombre de stations de mesure des gaz-critère en Suisse pour la période allant de 1992 à 1996 (Baehler EurJAC).

\begin{tabular}{lcccccc}
\hline Évolution 1992-1996 & $\mathrm{SO}_{2}$ & $\mathrm{NO}_{2}$ & $\mathrm{CO}$ & $\mathrm{O}_{3}$ & Poussières en suspension Poussières sédimentées \\
\hline Nombre total d'analyseurs & -32 & -8 & -13 & -18 & -25 & -24 \\
Variation moyenne absolue par service & -1.3 & -1.1 & -0.5 & -0.7 & -1.0 & -1.0 \\
Variation moyenne relative par service & $-32 \%$ & $-23 \%$ & $-30 \%$ & $-15 \%$ & $-46 \%$ & $-44 \%$ \\
\hline
\end{tabular}




\section{Épisode photochimique et modèles atmosphériques}

Il s'agit ici d'observer le développement d'un épisode photochimique, et de caractériser aussi précisément que possible sur une durée de quelques jours, en trois dimensions, l'évolution du réacteur photochimique atmosphérique. Un tel épisode a lieu en condition météorologique très favorable (condition anticyclonique, fort ensoleillement), avec des vents faibles, et un domaine d'étude dont l'extension spatiale contient le panache et la formation de polluants secondaires à simuler, soit une extension horizontale d'environ $100 \mathrm{~km}$ et verticale de quelques $\mathrm{km}$. Dans une étude d'épisode photochimique, chaque information de type météo ou chimie est valorisée : elle participe à la construction d'une base de donnée qui définit le « cas de base » ou le cas test de calage de modèles atmosphériques à mésoéchelle. Ces modèles sont constitués d'un ensemble de sous programmes qui reproduisent de manière prédictive la dynamique des polluants dans l'atmosphère à partir des émissions, de leur transport et diffusion induite par le vent, de leur transformation chimique, jusqu'à leur déposition par voie sèche ou humide, et ce en tenant compte du relief, du type de terrain, et bien sûr des sources d'émission présentes dans le domaine (cadastre d'émissions). Avec d'une part un cas de base bien décrit correspondant à une situation mesurée, et d'autre part un modèle eulérien mésoéchelle, les résultats simulés par le modèle sont directement confrontés aux mesures du cas de base. Partant d'une bonne représentation numérique de cette situation mesurée, le modèle permet de comprendre et d'analyser les résultats mesurés sur un épisode photochimique, et devient alors l'unique outil scientifique de contrôle, de manière prédictive, de l'efficacité de scénario de réduction de la pollution de l'air. Il est en fait le seul outil d'aide à la décision en matière de contrôle de la qualité de l'air.

À titre d'exemple de mesure d'un épisode photochimique, prenons la campagne de mesure effectuée en mai- juin 1998 proche de Milan, une étude dédiée en particulier à l'influence des émissions intenses de la grande agglomération milanaise en direction du nord sur la région lombarde, la plaine du Pô et le sud des Alpes suisses. À Seregno, à $20 \mathrm{~km}$ au nord de Milan, ont été installés un système Lidar Ozone (Quaglia, EurJAC) et un système de mesure de profils de vent (Ruffieux, EurJAC).

Ces deux appareils mesurent de manière simultanée, et la figure 3 présente une série temporelle de l'évolution de la formation d'ozone (3a) et de la vitesse et la direction des masses d'air (3b) à la verticale de Seregno. En combinant ces deux mesures, on obtient directement le flux d'ozone à chaque altitude et en fonction du temps, soit une mesure essentielle de test de la validité des modèles puisque combinant les effets d'une chimie complexe de formation de pollution secondaire avec une météorologie particulière à ce cas d'étude.

Sur la carte de la figure 4, le flux d'ozone est indiqué par les deux flèches à la verticale de Seregno, pour le cas du 13
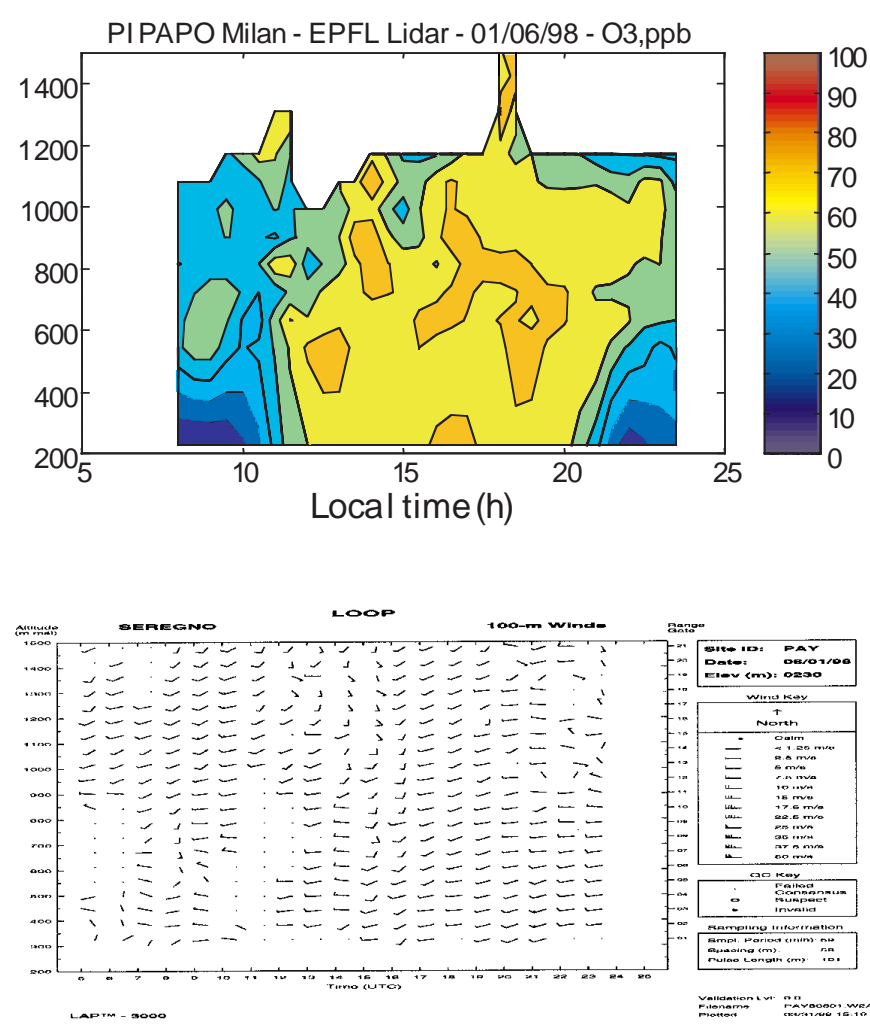

Figure 3. Série temporelle de mesure d'ozone par Lidar (Fig. 3a) (Quaglia, EurJAC) et de profils de vent par radar micro-onde (Fig. 3b) (Ruffieux, EurJAC) à la verticale de Seregno, le $1^{\text {er }}$ juin 1998.

mai à 16.30 à une altitude de $650 \mathrm{~m} / \mathrm{mer}$ et de $900 \mathrm{~m} / \mathrm{mer}$. À $650 \mathrm{~m} / \mathrm{mer}$ la masse d'air a une orientation ouest-est, alors qu'à $900 \mathrm{~m} / \mathrm{mer}$ elle est orientée sud-nord, cette mesure étant intégrée sur une durée de une heure $(16.40 \pm 30$ minutes $)$ et avec une résolution verticale de $100 \mathrm{~m}$. L'existence d'une telle stratification de masse d'air sur une région relativement plane est absolument inattendue. On réalise alors que de la vision simplificatrice d'un vent uniforme sud-nord sur toute la hauteur de la couche limite planétaire et qui entraînerait un panache de polluants émis depuis Milan de manière unidirectionnelle vers les Alpes Suisses n'est pas adaptée. Au contraire, il faut considérer maintenant le développement du panache de Milan à plus haute altitude, alors que proche de Seregno, l'orientation ouest-est de la masse d'air supprime l'influence directe de Milan. Notons qu'un tel cas de base, avec cette météorologie conduisant à des masses d'air stratifiées, est un " challenge » en tant que cas test des modèles à mésoéchelle !

Ces observations Lidar et de profils de vent sont liées à la « dynamique »du cas de base. Il s'agit d'autre part de déterminer les espèces chimiques qui sont en jeu dans cette étude. Alors que l'ozone est un polluant secondaire formé à partir des émissions d'oxydes d'azote $\mathrm{NO}_{x}$ et des composés organiques volatiles (COV), le contrôle de la formation 


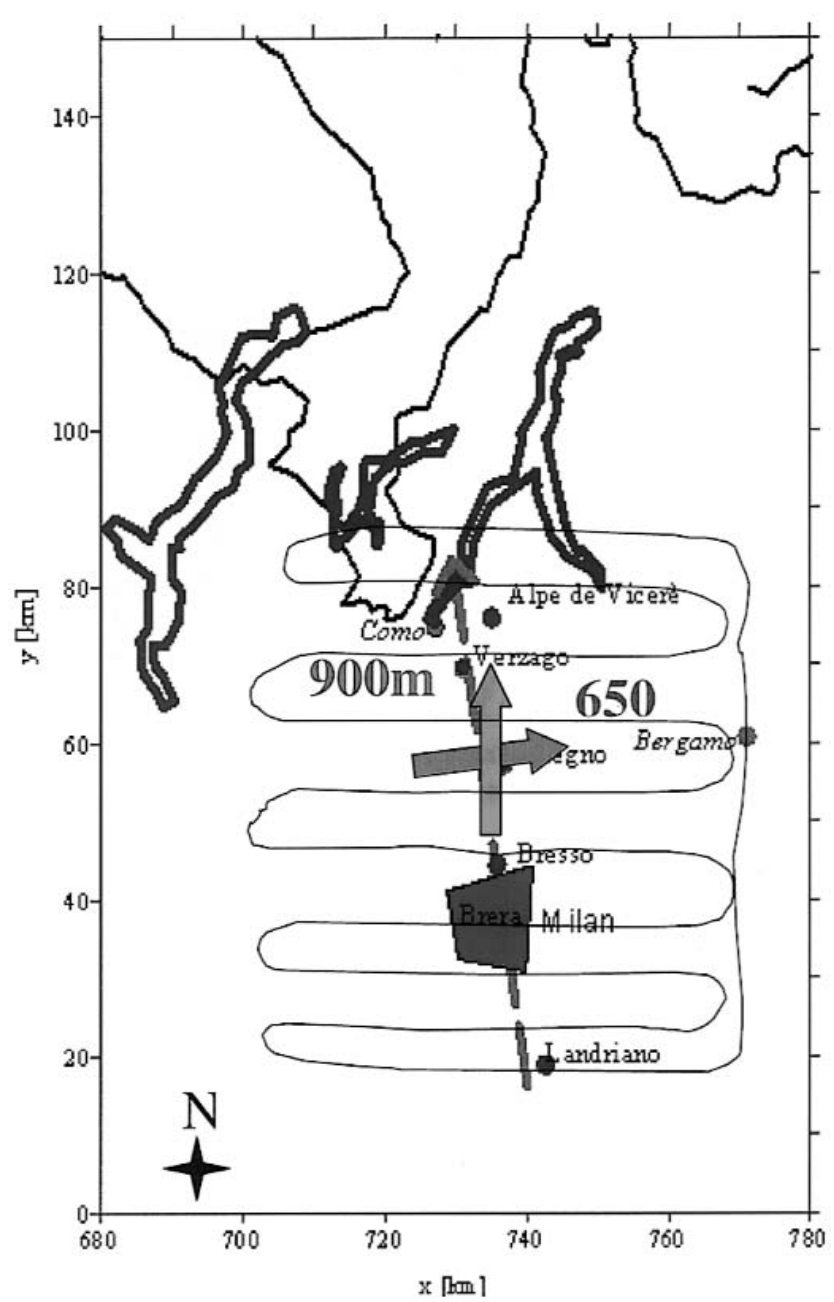

Figure 4. Carte du domaine d'étude de la campagne de mesure sur Milan, avec deux flèches indiquant respectivement le flux d'ozone à $650 \mathrm{~m} / \mathrm{mer}$ et à $900 \mathrm{~m} / \mathrm{mer}$, ainsi que la trajectoire de vol schématisée de l'ARAT (voir texte) et l'emplacement de divers autre sites de mesure (Quaglia, EurJAC).

d'ozone dépend de manière non linéaire des concentrations respectives de ces deux types d'émissions primaires. Cette observation a été un des résultats majeurs obtenus à partir des modèles atmosphériques et souligne le fait que réduire un type d'émission primaire n'implique pas forcément réduire la pollution secondaire.

\section{Tunnel d'évitement et augmentation d'ozone}

Afin d'illustrer ce point de manière simple, prenons l'exemple de l'ouverture récente du tunnel d'évitement de la ville de Sachseln en Suisse, cet ouvrage ayant eu pour conséquence une réduction de $50 \%$ du trafic en ville (Jimenez,
Tableau II. Comparaison relative de l'évolution de la qualité de l'air en ville de Sachseln avant et après l'ouverture d'un tunnel d'évitement de la ville (Jimenez, EurJAC).

AVANT APRES Changement relatif [\%]

\begin{tabular}{lccc} 
Trafic total [jour-1 & $16^{\prime} 191$ & $8 ' 223$ & $-49,2$ \\
Camions [jour $\left.^{-1}\right]$ & $1 ' 365$ & 541 & $-60,4$ \\
$\mathrm{PM}_{10}\left[\mu \mathrm{g} / \mathrm{m}^{3}\right]$ & 43,7 & 31,1 & $-28,9$ \\
$\mathrm{PAH}\left[\mathrm{ng} / \mathrm{m}^{3}\right]$ & 23,2 & 11,0 & $-52,5$ \\
$\mathrm{NO}_{x}[\mathrm{ppb}]$ & 48,0 & 27,2 & $-43,3$ \\
$\mathrm{O}_{3}[\mathrm{ppb}]$ & 15,8 & 21,3 & $+34,7$ \\
\hline
\end{tabular}

EurJAC). Une station de mesure locale a permis de caractériser la qualité de l'air dans cette agglomération avant et après l'ouverture du tunnel, et ceci alors que les conditions météorologiques étaient très semblables sur les périodes de comparaison choisies. Le résultat de cette observation analysée de manière purement statistique, avec des valeurs intégrées sur 5 jours avant et après l'ouverture du tunnel, est présenté dans le tableau II.

Le trafic de ville a été réduit d'un facteur deux, avec comme conséquence une réduction des émissions primaires, des $\mathrm{NO}_{x}$ et des particules suspendues dans l'air $\left(\mathrm{PM}_{10}\right)$. Par contre, dans ce même cas d'étude, la production d'ozone en tant que polluant secondaire a elle augmenté de plus de $30 \%$ après l'ouverture du tunnel d'évitement de la ville.

\section{Indicateurs de la formation d'ozone}

Les modèles atmosphériques nous ont permis non seulement d'expliquer ces effets non linéaires, mais de plus de définir de nouveaux « indicateurs » du contrôle de la formation d'ozone (Neftel, EurJAC). Parmi ces indicateurs, il y a la mesure de la concentration des $\mathrm{NO}_{Y}$ soit la somme des $\mathrm{NO}_{X}$ et des produits d'oxydation des oxydes d'azote. Sur la figure 4, la trajectoire de vol de l'ARAT est symbolisée, cet avion de recherche français du CNRS ayant effectué des vols à basse altitude dédiés à la mesure in situ du $\mathrm{NO}_{Y}$ (Perros, $E J A C)$ dans la couche de mélange, et à haute altitude de profils verticaux d'ozone à la verticale sous l'avion par Lidar (Quaglia, EurJAC). Au cours d'un vol dans la couche limite planétaire, la mesure de la concentration d'ozone et de $\mathrm{NO}_{Y}$ s'effectue dans des masses d'air très différentes, avec des masses définies comme «photochimiquement jeunes » proche des sources d'émissions primaires, soit proche de Milan, ou « photochimiquement âgées » lors des trajectoires de vol à proximité des Alpes Suisses.

Dans la figure 5, le potentiel de production d'ozone par $\mathrm{NO}_{Y}$ présent dans la masse d'air est une variable qui change selon " l'âge » photochimique de la masse d'air considéré. Cet âge photochimique est défini comme la quantité de $\mathrm{NO}_{X}$ 


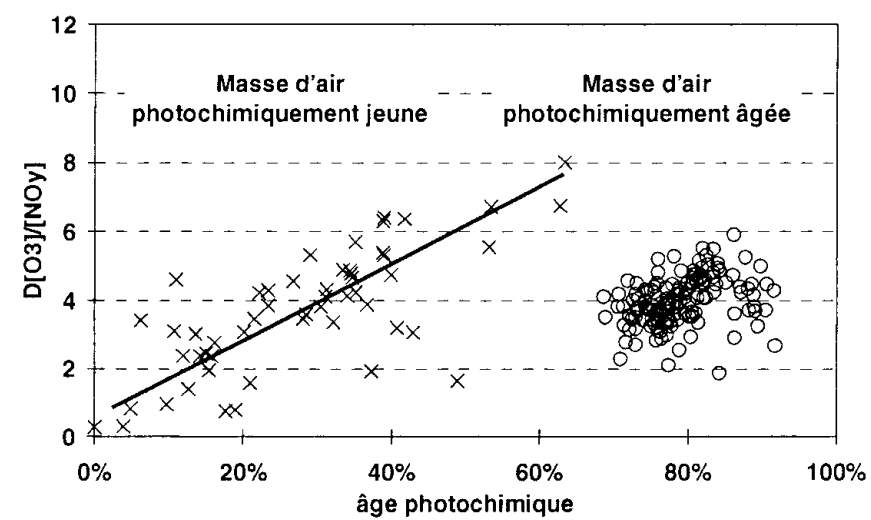

Figure 5. Potentiel de production de l'ozone par les $\mathrm{NO}_{Y}$ : dans une masse d'air photochimiquement jeune, il y a augmentation régulière de ce potentiel en fonction de la quantité de $\mathrm{NO}_{\gamma}$, alors que cette valeur reste constante (environ 6 molécules d'ozone par $\mathrm{NO}_{Y}$ ) pour une masse d'air photochimiquement âgée (Perros, EurJAC).

présents dans $\mathrm{NO}_{Y}$ : par exemple, $10 \%$ d'âge photochimique correspond à $10 \% \mathrm{NO}_{X}+90 \% \mathrm{NO}_{Z}=\mathrm{NO}_{Y}$. La production d'ozone augmente de manière linéaire dans une masse d'air jeune, pour atteindre une valeur de saturation de 6 molécules d'ozone produites par molécule de NO émise à $50 \%$ d'âge photochimique. Dans une masse d'air photochimiquement âgée (70 à $90 \%)$, il y a équilibre entre formation et destruction de l'ozone.

Notons la complémentarité entre mesures de type aéroportées et mesure au sol, les mesures effectuées à bord d'un avion donnant une image de l'extension spatiale du panache sur une durée de quelques heures, alors que les observations Lidar et de profileur de vent donnent sur un profil vertical l'évolution temporelle sur plusieurs jours de cet épisode.

Sur le même cas d'étude, mais plus au nord, à Verzago, ce sont d'autres indicateurs de la formation d'ozone qui sont testés à partir de mesures au sol de composés gazeux tels les $\mathrm{CH}_{2} \mathrm{O}, \mathrm{HNO}_{3}$ et $\mathrm{H}_{2} \mathrm{O}_{2}$ (Neftel, EurJAC).

\section{L’atmosphère « perturbée »}

Jusqu'ici, nous n'avons relevé que l'usage de méthodes de mesure en condition d'équilibre de l'atmosphère. Cependant certains gaz de la chimie atmosphérique se trouvent dans des concentrations qui rendent leur mesure à l'équilibre très délicate, alors qu'ils jouent un rôle clé dans la chimie de l'atmosphère. Ceci est valable en particulier pour le radical $\mathrm{OH}$ qui a la capacité d'oxyder les COV. Le radical $\mathrm{OH}$ étant extrêmement réactif, il a un temps de vie très court dans l'atmosphère, avec une concentration maximale inférieure à $10^{7} \mathrm{~mol} / \mathrm{cc}$ soit typiquement 5 ordres de grandeur plus faible que la concentration d'ozone. Afin d'apporter de nouvelles

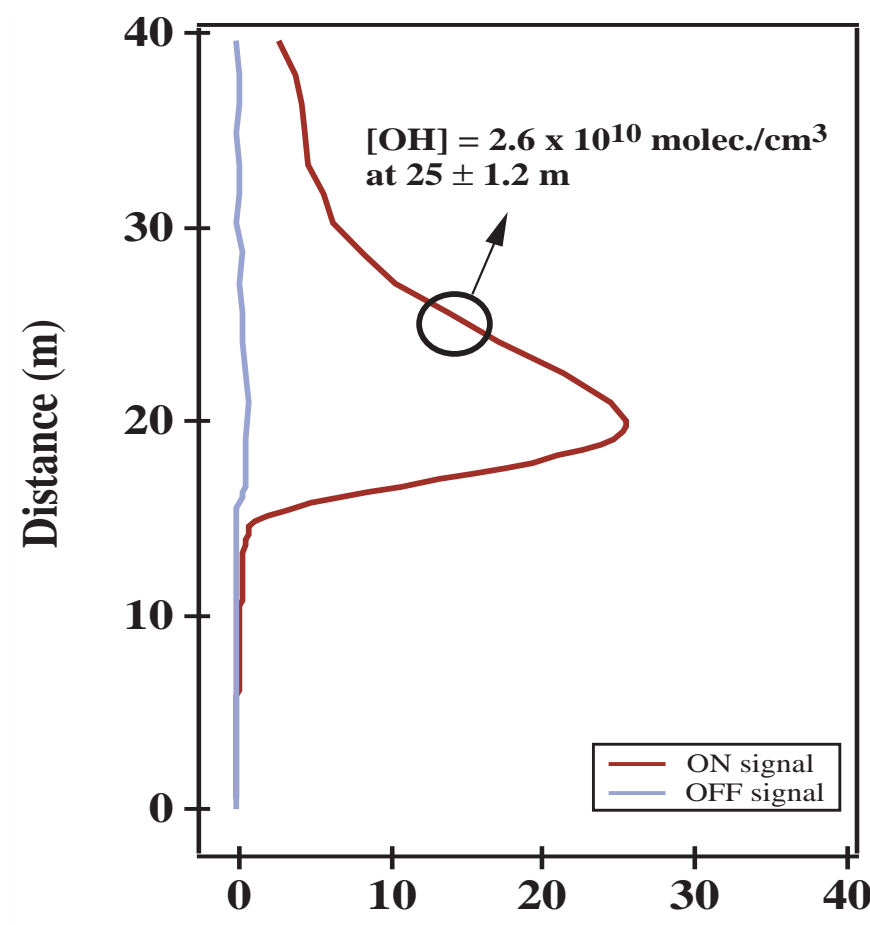

Range corrected signal (a.u.)

Figure 6. Mesure Lidar de la concentration de $\mathrm{OH}$ résiduelle par fluorescence induite (ON signal) une milliseconde après photolyse éclair de l'ozone et formation quasi-instantanée du OH. En variant ce délais, le temps de vie du $\mathrm{OH}$ peut être mesuré à diverses altitudes, soit dans des atmosphères chimiquement très différentes. Le signal OFF est la ligne de base de l'expérience (Calpini, EurJAC).

informations sur la cinétique de réaction du $\mathrm{OH}$ dans l'atmosphère, il est possible de perturber l'atmosphère en générant du $\mathrm{OH}$ par photodissociation induite par laser de l'ozone, et de mesurer alors la cinétique de relaxation du $\mathrm{OH}$, c'est-à-dire le retour de la concentration de $\mathrm{OH}$ à son état d'équilibre par fluorescence induite par laser. Le $\mathrm{OH}$ réagissant avec des temps de vie très différents selon que cette méthode est appliquée en atmosphère polluée ou propre, cette méthode permet en fait de retrouver les constantes de vitesse de réaction entre $\mathrm{OH}$ et composés organiques volatiles, et donne accès à un nouveau critère de contrôle de la formation d'ozone (Calpini, EurJAC).

La figure 6 présente une mesure du $\mathrm{OH}$ obtenue par cette méthode dite de «pump and probe Lidar », mesure effectuée sur une portée de quelques dizaines de mètres, et pour un délai de $1 \mathrm{~ms}$ entre le tir laser de production du $\mathrm{OH}$ (faisceau « pump ») et le tir laser de mesure de la concentration de $\mathrm{OH}$ (faisceau « probe »). Ces deux faisceaux laser étant alignés en dehors de l'axe optique du télescope de réception, ce n'est qu'après environ 20 mètres qu'ils entrent totalement dans le cône de détection du télescope. En excitant le $\mathrm{OH}$ 
sur une bande d'absorption (ON signal), la concentration effective du $\mathrm{OH}$ est obtenue. En changeant le délai entre tir laser de formation du $\mathrm{OH}$ et tir laser de mesure de sa concentration résiduelle, il est possible de suivre la cinétique de relaxation du $\mathrm{OH}$ à chaque altitude, et donc le temps de vie du $\mathrm{OH}$ dans différents types d'atmosphère. Cette mesure donne en fait un nouveau critère de contrôle de la formation d'ozone, ceci à partir de la connaissance de la vitesse totale de réaction des hydrocarbures avec le $\mathrm{OH}$.

\section{Mesures des particules dans l'air}

Seule la chimie homogène en phase gazeuse a été évoquée jusqu'ici. Force est de reconnaître qu'à ce jour, les efforts de modélisation sur la chimie hétérogène de type gaz-surface solide ou liquide sont encore relativement peu avancés. S'ajoute à cela le fait que la chimie hétérogène, du moins de jour, ne semble pas jouer un rôle crucial dans l'évolution de la qualité de l'air à basse altitude. Cependant la présence de particules dans l'air, et en particulier celle des aérosols de taille inférieure à quelques microns, affecte la santé des individus sur terre. À ce titre il faut considérer ces particules en suspension comme une forme de pollution atmosphérique au même titre que la pollution induite par les espèces gazeuses. Il paraît donc intéressant de suivre les développements instrumentaux récents sur la caractérisation des aérosols, en particulier en spectroscopie de masse et en microscopie électronique.

La spectrométrie de masse à deux lasers permet l'analyse chimique des composés aromatiques adsorbés sur des particules en suspension dans l'air. Après collection de ces particules sur un filtre (éthalomètre), l'échantillon est introduit dans une installation à vide, un premier tir laser infrarouge induisant une désorption efficace des aérosols du filtre, alors que le deuxième tir laser UV permet d'ioniser de manière sélective, en accordant la longueur d'onde du laser, un type de composés aromatique donné. La figure 7 présente la mesure du pyrène à la masse $202\left(\mathrm{C}_{16} \mathrm{H}_{10}\right)$ pour une prise d'échantillons effectuée toutes les 15 minutes en ville de Zurich le 3 février 1998 dans une rue à fort trafic (Haefliger, EurJAC).

La microscopie électronique offre un potentiel d'étude complémentaire des particules atmosphériques, et les développements récents en microscopie électronique à haute transmission et en spectroscopie de perte d'énergie apportent de nouvelles informations, par exemple sur les différents types de suie en suspension dans l'air. En combinant la microscopie électronique à balayage et à transmission, la particule est analysée de manière la plus détaillée, aussi bien en terme de morphologie (structure cristallographique, amorphe...) que de composition chimique.

La figure 8 montre l'exemple d'un échantillon contenant des particules inorganiques collectées en milieu urbain (Buffat, EurJAC). À faible grossissement (Fig. 8a) en microscopie à transmission, une vue «d'ensemble » des particules

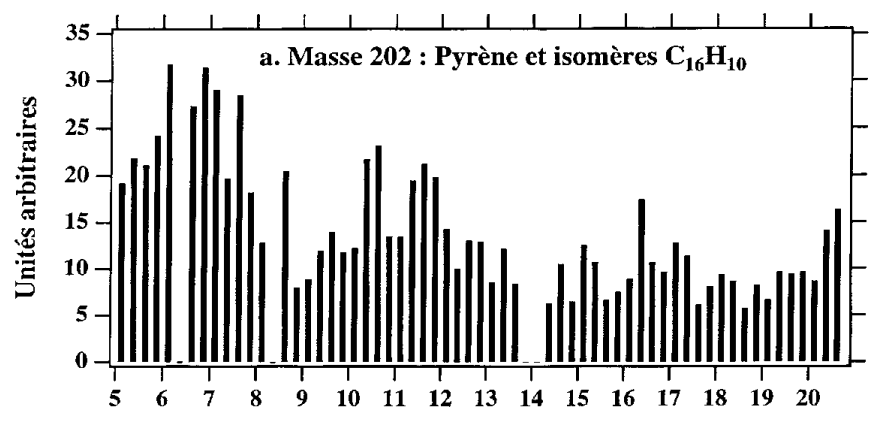

Figure 7. Série temporelle de mesure du pyrène en ville de Zurich à partir d'une analyse sous vide de spectroscopie de masse à deux lasers (Haefliger, EurJAC).

collectées est obtenue, alors qu'à fort grossissement (Fig. 8b), des aérosols de type fibre sont mis en évidence. L'image de diffraction électronique (Fig. 8c) de cet échantillon avec un pas de diffraction de $0,53 \mathrm{~nm}^{-1}$ permet d'identifier la structure cristallographique spécifique de ces fibres, alors que leur composition chimique (Fig. 8d) est donnée par spectroscopie de dispersion d'énergie par rayons $\mathrm{X}$, l'ensemble de ces investigations ayant été obtenues dans la même chambre d'analyse.

\section{Mesures de type semi-conducteur}

Pour revenir aux mesures des gaz critères, rappelons que les techniques utilisées, principalement de type mesure optique (absorption optique différentielle pour l'ozone, chimiluminescence pour les $\mathrm{NO}_{X}$, fluorescence induite pour le $\mathrm{SO}_{2}$ ou mesure photoélectrique pour les hydrocarbures polycycliques aromatiques, par exemple) requierent des appareils de mesure relativement coûteux. Arrivent sur le marché de nouveaux appareils de mesure basés sur l'application de capteurs de type semi-conducteur avec des performances (limite de sensibilité, stabilité) acceptables pour les mesures à l'imission. À titre d'exemple, le monoxyde de carbone peut être mesuré par réaction électrochimique en présence d'une électrolyte solide de Nafion, induisant un courant sur une électrode de diffusion téflon-platine, courant linéairement proportionnel à la concentration ambiante de $\mathrm{CO}$ (van der Wal, EurJAC). Cette mesure se caractérise par une bonne sélectivité au $\mathrm{CO}$, une limite de sensibilité inférieure à $0,5 \mathrm{ppm}$, et une excellente stabilité à long terme, ce qui est illustré à la figure 9, pour une mesure sur 1200 jours avec une concentration de référence stabilisée à $1 \mathrm{ppm}$ de $\mathrm{CO}$. De tels capteurs sont des alternatives intéressantes de mesure « à coût réduit » de gaz critères dans l'atmosphère. 

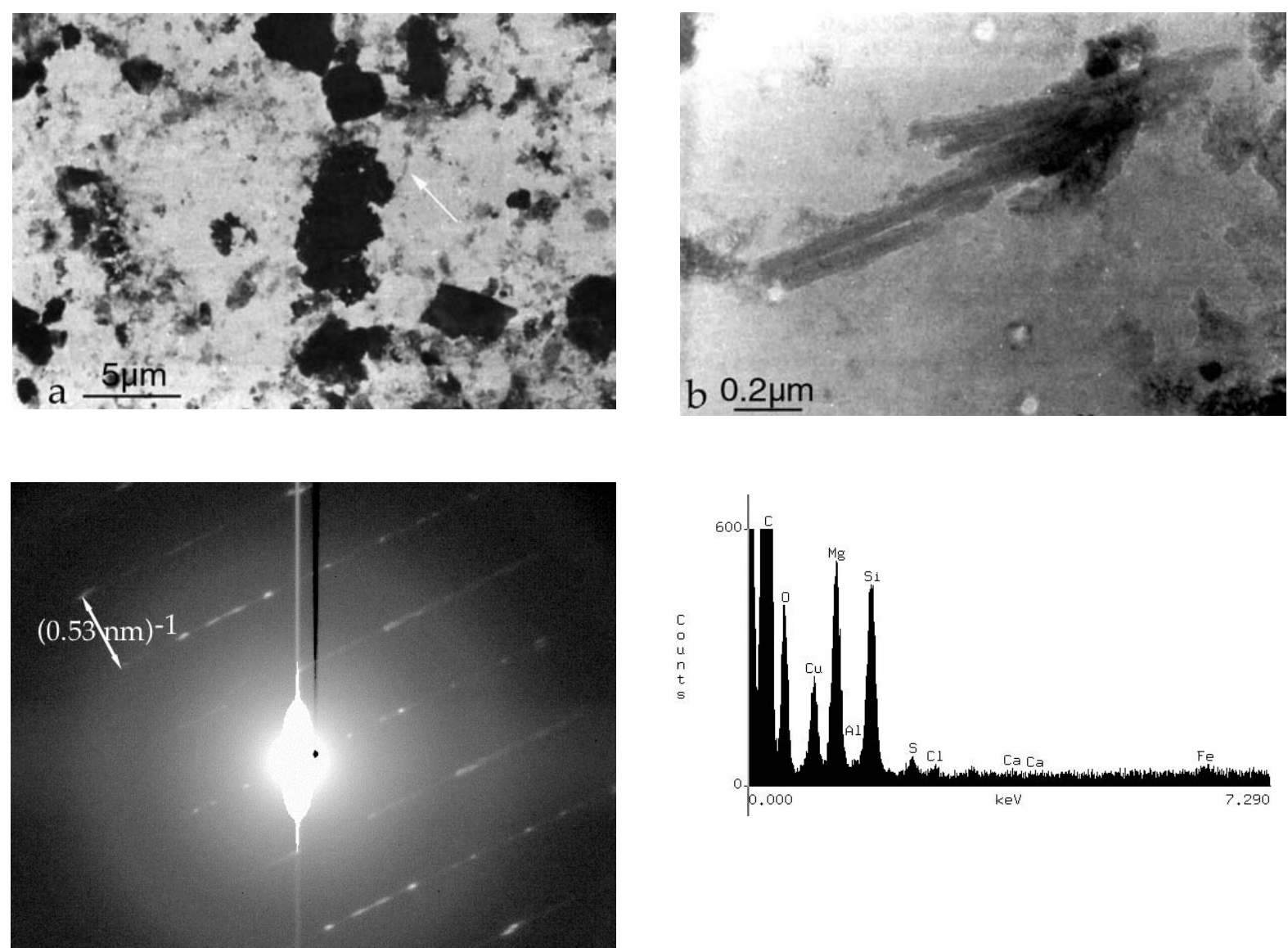

Figure 8. Analyse par microscopie électronique d'un échantillon de particules atmosphériques avec présence de fibre. Cette figure illustre diverses étapes d'analyse de l'échantillon obtenues dans la même installation, avec " l'observation directe " des particules par microscopie électronique à transmission (faible grossissement (a) et fort grossissement (b)), et la mesure par diffraction électronique (c) et par mesure RX (d) (Buffat, EurJAC).

\section{Pollution « indoor »}

Alors que l'homme est soumis en atmosphère libre à diverses sources de pollution de l'air pouvant affecter sa santé, il subit une atteinte supplémentaire en « atmosphère conditionnée » dans les bâtiments dans lesquels il travaille et vit. À titre d'exemple, le radon est un gaz radioactif provenant des traces d'uranium présentes dans le sol à l'état naturel, un gaz qui ne présente pas de danger de par sa trop faible concentration en atmosphère libre, mais qui, lorsqu'il est confiné dans des bâtiments peu ventilés, peut atteindre des seuils critiques, ceci d'autant plus que certains matériaux de construction sont de forts émetteurs de radon. La concentration de radon s'obtient par adsorbtion sur du charbon actif durant une période de quelques jours, puis dilution dans une solution de toluène, et mesure par méthode de scintillation (Buchillier, EurJAC). Une étude détaillée sur les concentra-

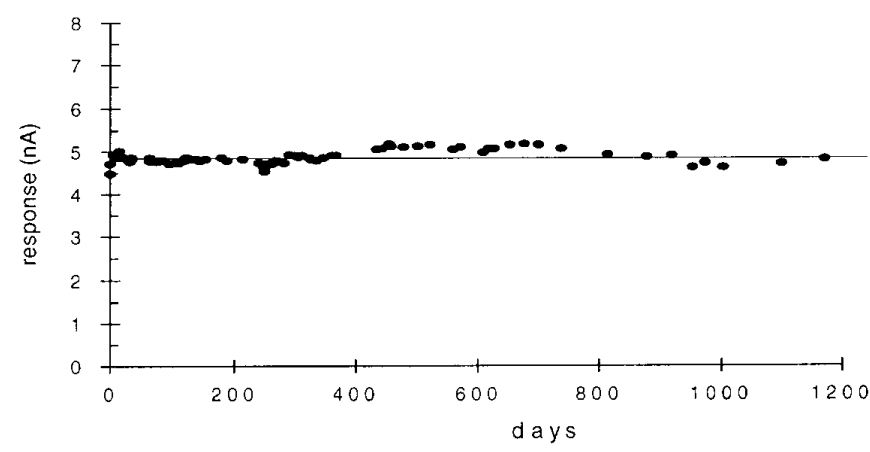

Figure 9. Mesure de la concentration de monoxyde de carbone par capteur semi-conducteur à base de Nafion, sur une durée de 1200 jours, pour une concentration de CO stabilisée à 1 ppm (van der Wal, EurJAC). 


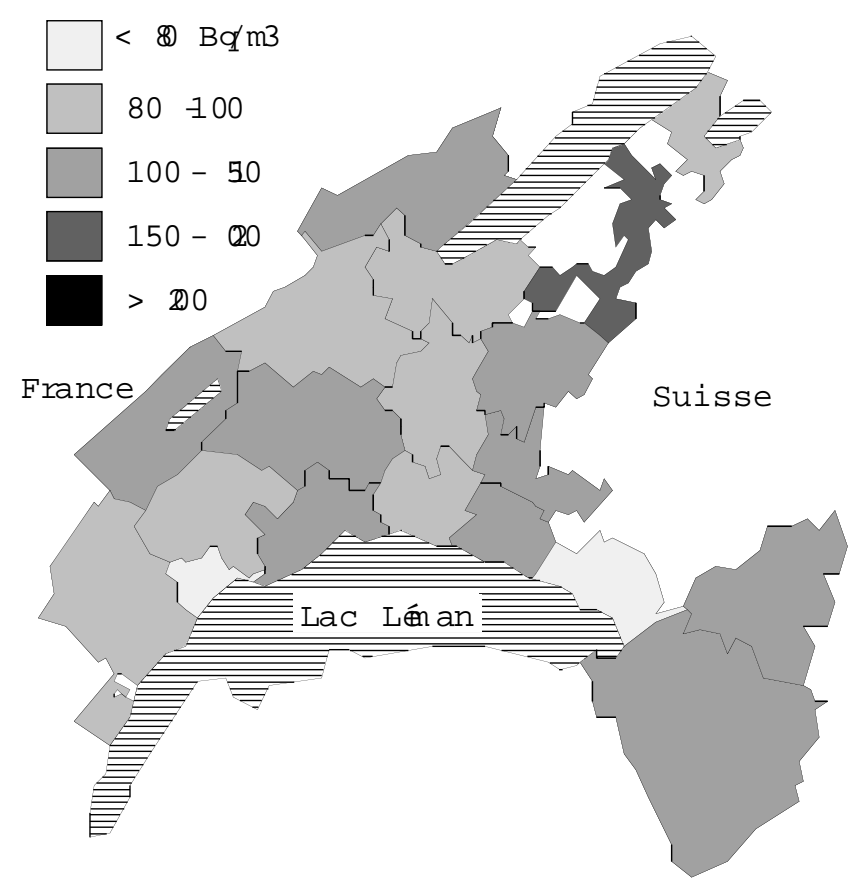

Figure 10. Répartition de la concentration moyenne de radon par région, sur la carte du Canton de Vaud en Suisse. Cette répartition est obtenue à partir d'un échantillon de 3000 bâtiments dans lesquels cette mesure a été effectuée de décembre 1997 à mai 1998 (Buchillier, EurJAC).

tions de radon dans les habitations du canton de Vaud en Suisse est en cours. Sur la carte de la figure 10, la distribution de concentration de radon dans les habitations est obtenue à partir d'un échantillon de 3000 bâtiments mesurés durant l'hiver 97/98, période durant laquelle les concentrations de radon sont les plus élevées. Les concentrations moyennes sont de l'ordre de $100 \mathrm{~Bq} / \mathrm{m}^{3}$, alors que la limite en Suisse pour les nouvelles habitations est fixée à $400 \mathrm{~Bq} / \mathrm{m}^{3}$. Néanmoins $5 \%$ des bâtiments investigués dépassent cette norme.

\section{Impact de l'ozone sur notre environnement}

Alors que les effets de la pollution de l'air sur l'homme sont difficilement dissociables des autres atteintes que nous subissons (tabagisme, comportement nutritionnel, ...) le monde végétal offre une possibilité de détection ciblée de ces impacts. Prenons pour exemple les études d'impact de l'ozone sur la végétation, qui ont pour but de déterminer les mécanismes d'attaque de l'ozone sur la végétation, mais aussi la réponse de diverses cultures à des doses variables d'ozone.

La Dose Absorbée de Polluant (DAP) est le critère de mesure, un critère qui dépend du transport atmosphérique de

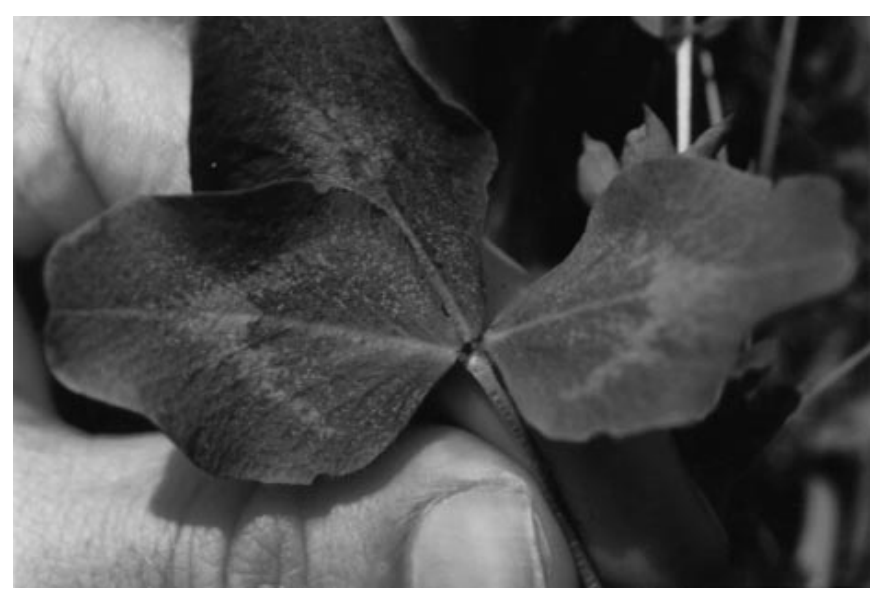

Figure 11. Observation de nécroses (nombreuses petites taches blanches sur les feuilles) dues à la présence de fortes concentrations instantanées d'ozone sur un trèfle rouge. Plante récoltée à Cadenazzio en mai 1998 durant LOOP (Fuhrer, EurJAC).

l'ozone par diffusion turbulente, de sa diffusion moléculaire à la surface de la plante et enfin de sa diffusion moléculaire dans les pores de la plante, ce dernier paramètre jouant un rôle majeur et contrôlant essentiellement la DAP.

Pour l'ozone, on peut séparer son impact sur les effets à courte durée, caractérisés par une DAP élevée sur quelques heures, des effets à longue durée (Fuhrer, EurJAC). La figure 11 montre l'exemple d'une feuille de trèfle rouge avec symptômes typiques de nécroses dues à l'ozone. Cette plante a été récoltée là où les plus fortes concentration d'ozone ont été mesurées durant la campagne de Milan, soit au sud du Tessin dans la région de Cadenazzio, avec des concentrations d'ozone dépassant $400 \mu \mathrm{g} / \mathrm{m}^{3}$.

Alors que cette photo illustre un effet observé dans le terrain, il est possible d'étudier l'impact de l'ozone en utilisant des serres dans lesquelles l'air est généré de manière synthétique, et d'observer par exemple comment l'ozone affecte la production de grains de blé. La figure 12 présente les résultats de diverses études effectuées en Europe et aux États-Unis sur la production relative de grains de blé en fonction de la DAP, $100 \%$ de production représentant le rendement obtenu en absence d'ozone, alors que l'index AOT40 exprime la concentration d'ozone accumulée en heures pour lesquelles la concentration d'ozone a dépassé $40 \mathrm{ppb}$ (Fuhrer, EurJAC). Ce résultat très important montre qu'en dessous d'une AOT40 de $3 \mathrm{ppm}$ heure, la production reste inchangée, alors que pour une valeur 10 fois plus élevée, ce rendement agricole est divisé par deux ! C'est à partir de ce résultat que la valeur de $3 \mathrm{ppm}$ heure, calculée sur une période de 3 mois, a été fixée comme le niveau critique de protection la production du blé en Europe.

En mettant en évidence la relation directe entre production agricole et surconcentration d'un polluant 


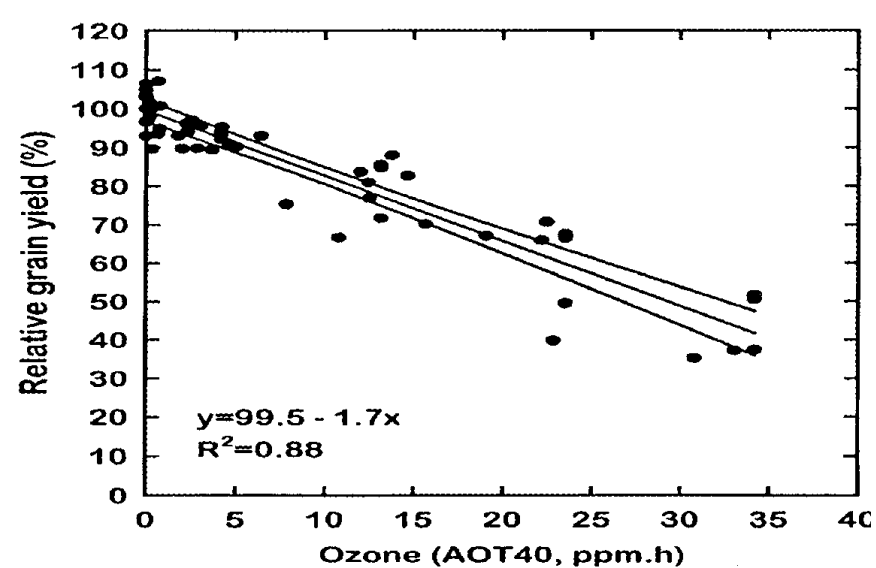

Figure 12. Rendement relatif de la production de grains de blé en fonction de la concentration accumulée d'ozone en heures AOT40 de plus de $40 \mathrm{ppb}$, et ce sur une période de trois mois (Fuhrer, EurJAC).

atmosphérique, on réalise tout l'enjeu économique qui est lié à la pollution de l'air. Cependant, une analyse détaillée de cet impact peut révéler une grande variabilité dans le résultat sur la productivité de divers végétaux. La figure 13 résume pour deux espèces végétales différentes l'impact de l'ozone sur la production de la biomasse de ces plantes, cette biomasse étant réparties selon : les feuilles/les tiges/et les fleurs, et cette analyse étant reportée pour une atmosphère sans et avec ozone. Pour l'Achillea millefolium, la présence

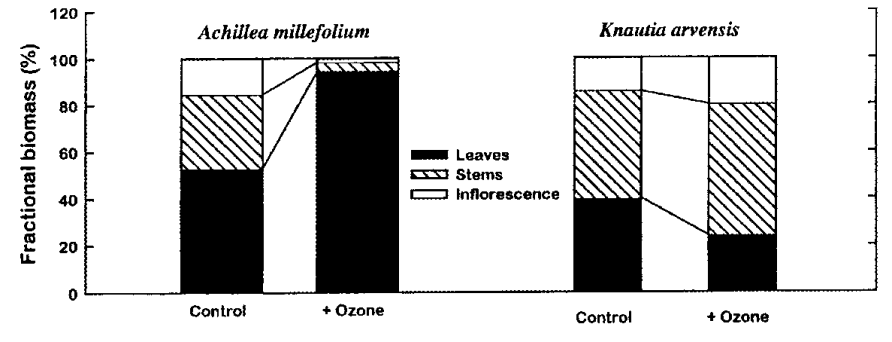

Figure 13. Développement de la biomasse, pour Achillea millefolium et Knantia avensis, avec et sans ozone (Fuhrer, EurJAC).

d'ozone stimule une forte croissance du feuillage, alors que pour la Knantia avensis, c'est un effet exactement opposé qui est observé.

\section{Remerciements}

L'auteur de ce papier tient à remercier chaleureusement l'ensemble des « acteurs » de cette conférence GACHI à l'EPFL en automne 1998, pour leur contributions orales et pour les différents articles publiés dans le Dossier «Air Pollution : Measuring techniques and impacts on our environment » dans EurJAC. Pour les références et les explications détaillées des divers aspects présentés ci-dessus, le lecteur voudra bien se reporter à ce Dossier. 